\title{
Mobility of Students, Academic and Administrative Staff: A Basis for Establishing a European Higher Education Area (Berlin Communiqué 2003)
}

\author{
Hilde Chantrain \\ Department of Speech-Language Therapy, Lessius Hogeschool, Antwerp, Belgium
}

\section{Key Words}

Intensive Programme • Lifelong Learning Programme •

Speech-language therapy

\begin{abstract}
This article aims to highlight the possibilities of the Intensive Programme (IP), one of the items of the Lifelong Learning Programme of the European Commission. The benefits of organizing an IP in a specific discipline-related area is illustrated by a case study of the speech-language therapy IP, which has been coordinated for 16 years by the Department of Speech-Language Therapy of the Lessius Hogeschool Antwerp, Belgium.

Copyright $\odot 2010$ S. Karger AG, Basel
\end{abstract}

\section{Introduction}

The Erasmus Programme creates a wide range of opportunities for individuals to study, work, teach or be trained in another European country. Apart from an improved command of other languages and deeper insights into the host countries' cultures, students and teachers can acquire knowledge in new subject areas or teaching methods, and improve their organizational skills as well as their intercultural understanding in multicultural Europe.
One of the possibilities to promote and increase Erasmus mobility in all kinds of study areas is the Intensive Programme (IP), which actually is a substantial part of the new Lifelong Learning Programme (2007-2013).

\section{What Is an IP?}

An IP is a short programme of study, ranging from 10 days to 6 weeks of subject-related work, bringing together students and staff of different higher education institutions (HEI) from at least 3 participating countries in Europe. The aim is to encourage and stimulate efficient and multinational teaching of specialized topics in a European dimension which otherwise might not be taught in regular programmes of studies at the respective home institutions. By working together in multinational groups, participants benefit from special learning and teaching settings and conditions which are not available in a single institution. Furthermore, students gain new perspectives on specific topics. Last but not least, teaching staff members can exchange views on their teaching contents and new curricular approaches as well as test teaching methods in an international (classroom) environment.

An IP can be either a one-off activity or an activity repeated over a limited number of years. The maximum duration of funding in this Erasmus programme is 3 con-

\section{KARGER \\ Fax +4161306 1234 \\ E-Mail karger@karger.ch}

www.karger.com
(C) 2010 S. Karger AG, Basel

$1021-7762 / 10 / 0625-0234 \$ 26.00 / 0$

Accessible online at: www.karger.com/fpl
Hilde Chantrain

Isabella Brantstraat 20

BE-2018 Antwerp (Belgium)

Tel. +32323759 98

E-Mail hilde.chantrain@skynet.be 
secutive years, although annual applications are needed. On behalf of all the participating project partners, the coordinating HEI submits the IP application to the national agency in the home country. All participating HEI must hold a valid Erasmus University Charter.

Against the background of the Bologna process, an IP particularly addresses the following challenges:

- stimulating cooperation with host countries which are traditionally less attractive;

- enabling transnational mobility periods both for students and teaching staff in subjects with no or little tradition of mobility periods;

- enabling less privileged target groups such as students in special socioeconomic situations to go abroad at least for a short period;

- implementing mobility periods for the 3-year Bachelor programmes (generally, it is more difficult to realize longer mobility periods due to the compact and tight programme structure).

\section{European Dimension of an IP}

The European dimension of an IP can be viewed from various angles. Firstly, from the perspective of mobility, an IP is an important tool for promoting and supporting mobility. This is especially the case with those students which, for whatever reason, are unable to go abroad for longer Erasmus study periods. Secondly, IPs further a subject-specific approach, i.e. IPs can increase European cooperation within a certain discipline or study area. Moreover, IPs are interesting from an academic point of view, as they give students and teachers from different European countries the possibility to discuss their own subject.

To represent a discipline-specific organization is an enormous advantage. Actually, it is of crucial importance that each IP is embedded within institutional policies. The expected long-term effect is to change approaches to curricula, teaching and learning. Furthermore, an IP has to be recognized as part of the regular curriculum by allocating an agreed number of ECTS (European Credit Transfer and Accumulation System) credits to all participants. And last but not least, an IP can also be a powerful tool in any policy an institution may have for its internationalization at home.

The following case study seeks to highlight the benefits of organizing an IP, and to exemplify this in a specific discipline-related area of educational programmes, namely in speech-language therapy (SLT).

\section{Case Study: SLT IP}

History

The SLT IP was established in 1993 by a group of 7 (Western) European HEI coordinated by the Lessius Hogeschool. From then on, the consortium has continuously expanded in order to bring 'new' and more extensive European expertise and students into the programme. This expansion resulted in a present-day consortium of 18 participating HEI:

- Antwerpen Lessius Hogeschool (3-year BA programme)

- Haute Ecole Leonard de Vinci Bruxelles (3-year BA programme)

- Katholieke Universiteit Leuven (4-year MA programme)

- Katholieke Hogeschool Brugge-Oostende (3-year BA programme)

- Hanzehogeschool Groningen (4-year BA programme)

- Göteborg Universitet (5-year MA programme)

- Karolinska Institutet Stockholm (5-year MA programme)

- Universitat Autònoma de Barcelona (3-year BA programme)

- Oulun Yliopisto (University of Oulu; 6-year MA programme)

- Université Louis Pasteur Strasbourg (4-year BA programme)

- Universidad de Valladolid (3-year BA programme)

- Trinity College Dublin (4-year B.Sc. Honours Programme)

- Università degli Studi di Padova (3-year BA programme)

- University of Strathclyde Glasgow (4-year B.Sc. Honours Programme)

- Rheinisch-Westfälische Technische Hochschule (RWTH) Aachen (5-year MA programme)

- Fontys Paramedische Hogeschool Eindhoven (4-year BA programme)

- University of Malta (4-year B.Sc. Honours Programme)

- FH Joanneum Graz (3-year BA programme)

This group of HEI organized IPs for SLT students annually, for 2 weeks and in different locations such as Aachen, Amsterdam, Cardiff, Brussels, Groningen, Gothenburg, Padua, Glasgow, Dublin, Valladolid, Malta, Oulu, Strasbourg, Eindhoven, Barcelona and Stockholm. 


\section{Content}

This broad consortium, with representatives from all corners of Europe, has facilitated the interaction between a diversity of countries and research cultures, which has proven to be an enriching experience for all involved. The discipline-related content of all these programmes offers a more comprehensive curriculum to all participating HEI, but also enables students to gain more in-depth knowledge and broaden their view on their own discipline.

\section{Specific Themes}

The theme of each SLT IP is agreed on by consensus by all members of the consortium during the annual general meeting of all partners. Choosing different themes allows the IP to combine a content-related focus and a European dimension. Themes of past IPs are listed below:

- IP on aphasia and stuttering (1993-1994)

- IP on language disorders and hearing disorders (19941995)

- IP on voice disorders and augmentative communication (1995-1996)

- IP on reading disorders and motor speech disorders (1996-1997)

- European management in SLT (1997-1998)

- Multidisciplinary approach in SLT (1998-1999)

- Speech and language impairments: a European management programme (1999-2000)

- SLT: towards an international dimension in assessment and therapy (2000-2003)

- Building bridges between research, assessment and therapy in European SLT programmes (2003-2006)

- Moving towards harmonization of SLT programmes in the European context (2006-2009)

\section{General Project Description}

This IP is a discipline-related project, geared towards SLT students of 18 educational programmes from $12 \mathrm{Eu}-$ ropean countries. The IP relates, complementarily as well as supplementarily, to the regular curricula of the participating departments. Students and staff work together during 12 days on specialized topics of the field. Bringing so many students and staff together adds not only a pedagogical, but also an international surplus value to their training.

The IP is organized yearly at various partner HEI. Its content focuses on new research-based papers by all the participating institutes and on specific seminars. All research-based papers and seminars are given by staff of the different institutes. Master students present their re- search projects. Seminar topics are chosen on the basis of and related to the strengths of the participating institutes.

After a written report, each student is credited. All students and staff receive a certificate of attendance at the end of the programme. During the programme, the students participate in an interactive way with staff and other students. This gives them a great opportunity to expand their knowledge in the SLT field even in an intercultural context. It lends their individual training a strong European dimension. Staffs also have a vast experience, and this intensive cooperation gives them the opportunity to move towards a harmonization of SLT programmes in Europe.

\section{Pedagogical and Didactical Approach}

An IP enables students to meet and work with students coming from various countries. They also have the chance to meet academic experts from different countries, disciplines and academic backgrounds. Moreover, they are confronted with various academic traditions, scientific perspectives and teaching methods in a multinational and multicultural surrounding. An IP should provide something new in terms of learning opportunities, skill development and access to information, for the participating teachers as well as for the students.

By bringing together experts from numerous partner universities, students also profit from this excellent teaching potential. Academics, in turn, have the opportunity to discuss their curriculum in an international environment, test new teaching methods and, in the long term, might form a network to develop joint modules or even joint programmes. The 12-day IP is mostly based on a combination of lectures, research-based papers, workshops, seminars, student presentations and working visits related to a wide range of SLT topics. The common language of the programme is English.

The academic staff of all partner universities is responsible for the lectures and interactive seminars. Key lectures and research-based papers are presented in plenary sessions, whereas seminars and workshops are held in small groups to enlarge the interactivity. During the student presentation, all groups of students present their own SLT curriculum. A course book of the whole programme is provided to all students (paper version and/or CD ROM).

\section{Selection and Evaluation Procedures}

Prior to any IP, a student selection takes place at each participating institute following commonly agreed criteria. These include: successful completion of their first 
year of study; proficiency in spoken and written English, and motivation for a mobility experience.

The evaluation of the project takes place at different levels. Students evaluate the content, the teaching method and interactive approach of each lecture/seminar. At the end of the programme, a general evaluation takes place with regard to the content and organizational aspects of the project.

\section{Dissemination Procedures}

Possibilities to disseminate the results of the project to a broader audience are taken into consideration. After each IP, all participating students inform their fellow students about the impact and the advantage of this programme. All student groups publish an article on the IP in their local student magazines. A special area of the website http://www.lessius.eu/IP has already been established to disseminate information on an IP worldwide. All abstracts of the courses and seminars are available on the website. This website is also used as a forum for communication prior to and during the programme. Some teaching establishments request a report from each student related to the scientific and social aspects of the programme; this would be part of the assessment mode for awarding ECTS credits for the participation in the IP. On a regular basis new partners are invited to participate in the project. So the network is growing continuously.

\section{Financial Aspect of IPs}

Erasmus IPs are financed by the European Commission as part of the Lifelong Learning Programme. In order to encourage more and more countries to organize this kind of programme, the financial support has been decentralized to each country's national agency. The EU commission distributes the money proportionally to each country.

The financial support is restricted to 60 students and 20 staff members, and 3 aspects of the programme are financed: organizational costs as a lump sum; travel costs of teachers and students at $75 \%$ of the real cost rate, and subsistence costs of teachers and students in daily rates related to the costs of living in each country.

The contribution by the EU does not cover the complete costs of the programme. Thus, all participating HEI contribute financially to the successful implementation of the programme.

\section{Success Story}

In 2008, the European Commission published a list of success stories regarding the organization of IP in the previous 5 years. The SLT IP described above has been selected as one of these success stories.

Following their long-lasting cooperation and collaboration via the IP, some partners are now jointly involved in other Erasmus projects, namely Curriculum Development Programmes. Two of these are in progress: (1) the European Clinical Specialization Course in Fluency Disorders, and (2) the European Clinical Specialization Course in Neuro-Motor Disorders. Other universities/ partners are increasingly expressing their interest in joining these EU programmes, or in writing up other proposals for joint participation. 